TAO, Vol. 12, No. 3, 537-558, September 2001

\title{
Solar Wind Streamlines in the Magnetospheric Noon-Midnight Plane
}

\author{
Tyan Yeh $^{1, *}$
}

(Manuscript received 11 May 2000, in final form 21 May 2001)

\begin{abstract}
The magnetosphere formed in the interaction between the magnetized solar wind and the earth's magnetic field has a magnetopause that separates interplanetary and terrestrial plasmas. The self-closed magnetopause is covered by the solar wind streamlines that are closest to the earth. Solar wind streamlines are delineated by intersection curves of electric and magnetic equipotential surfaces. The front part of the magnetopause is an electric equipotential surface that interfaces between oppositely directed interplanetary and closed field lines. The rear part is a magnetic equipotential surface that has open field lines passing through. Thus the magnetopause current is intense on the former closedness part of the magnetopause, which is a tangential discontinuity, and insignificant on the latter openness part, which is a rotational discontinuity. The part of closedness magnetopause has a pair of T-type magnetic neutral points on the exterior/interior face of the current sheet. The part of openness magnetopause has an X-type magnetic neutral line, in the studied case of southward interplanetary field. The neutral line is manifested by its cross point in the noon-midnight plane in our two-dimensional elucidation. In the noon-midnight plane, which is an electric equipotential surface, solar wind streamlines are delineated by traces of magnetic equipotential surfaces.
\end{abstract}

(Key words: Solar wind streamlines, Magnetopause current, Closedness magnetopause, Openness magnetopause,

Partially open magnetosphere)

\section{INTRODUCTION}

Formation as well as sustainment of the earth's magnetosphere in the continual interaction between the magnetized solar wind and the earth's magnetic field is a complicated magnetohydrodynamic process. It involves not only the diversion of the solar wind flow by the earth's magnetic field but also the reconnection of the interplanetary field lines with the terres-

\footnotetext{
${ }^{1}$ Institute of Space Science, National Central University, Chung-Li, Taiwan

${ }^{*}$ Corresponding author address: Prof. Tyan Yeh, Institute of Space Science, National Central University, Chung-Li, Taiwan 32054; E-mail: tyeh@ jupiter.ss.ncu.edu.tw
} 
trial field lines in a quasi-steady manner. The result is a finite-volume cavity that excludes the solar wind from getting closer to the magnetized earth. This magnetospheric cavity is filled with terrestrial plasma. It constitutes the magnetosphere proper, the boundary surface of which is the magnetopause in entirety. Thus the magnetopause, interfacing between the interplanetary and terrestrial plasmas, is covered by the solar wind streamlines that come in contact with the terrestrial plasma.

Following Chapman and Ferraro's pioneering work, the early theories of the magnetosphere were built on variant models of a completely closed magnetosphere (see Chapman

(a)

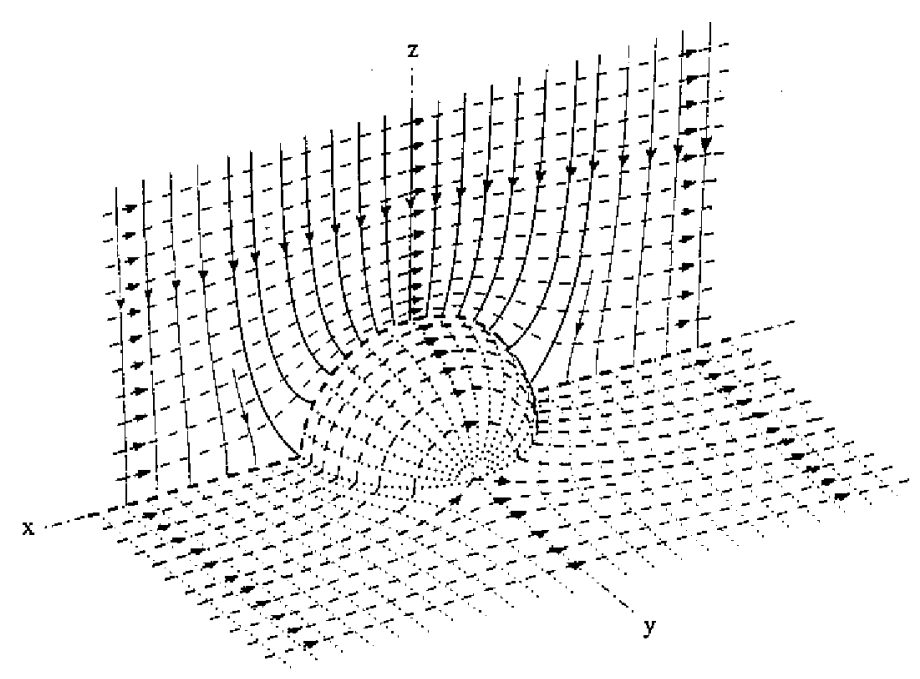

(b)

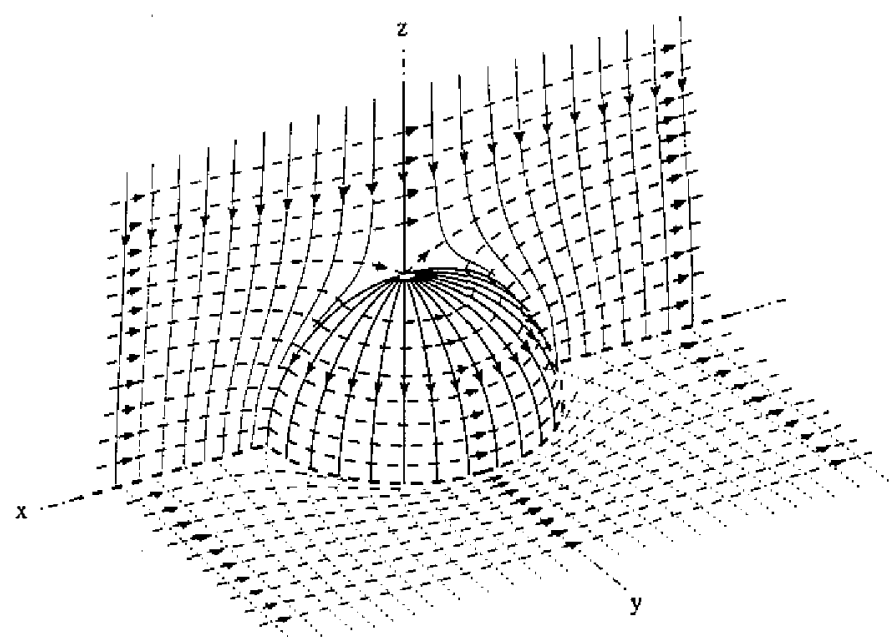

Fig. 1. Solar wind streamlines around a spherical magnetopause ( $\boldsymbol{a}$ ) in a closed magnetosphere and $(b)$ in an open magnetosphere. Solid lines are magnetic field lines and dotted lines are electric field lines. Dashed lines are streamlines. 
1963). Many later theories were to model a fully open magnetosphere after Dungey's suggestion of magnetic reconnection at the magnetopause (Dungey 1961). For a comprehensive review on these various models see Siscoe (1988). However, spacecraft traversings of the magnetosphere have shown that the earth possesses not only a current-filled magnetopause with cusp structures on the day side (see Cahill 1995) but also a magnetotail with open field lines on the night side (see Ness 1987). Thus, on the quasi-steady and quasi-global scales the earth's magnetosphere is neither completely closed nor fully open. It is a partially open magnetosphere, closed in the front and open in the rear.

The incident flow of the solar wind is diverted by the intense magnetopause current on the magnetopause's front part, which acts as a barrier to the impinging solar wind. The magnetopause' rear part is passed through by open field lines, which connect the earth magnetically to interplanetary space. Hence, the front part of the magnetosphere is like a closed magnetosphere and the rear part like an open magnetosphere.

The magnetopause of a partially open magnetosphere has a closedness part in the front and an openness part in the rear. The closedness magnetopause is an electric equipotential surface and the openness magnetopause is a magnetic equipotential surface. In this paper we shall educidate the above-mentioned magnetopause structure by a two-dimensional treatment. The obtained results reveal the noon-midnight meridional features of a corresponding threedimensional magnetosphere. It should be noted that the diverted flow of the solar wind is inherently three-dimensional (see Fig. 1). Global continuity may require some of the segmental planar streamlines in the noon-midnight plane to be connected by off-plane streamlines. The necessitated off-plane diversion is accounted for by sink and source of mass and flux flows in the two-dimensional context.

\section{MAGNETOSPHERIC MAGNETIC FIELD}

Consider a solar wind plasma that moves toward the earth with a velocity

$$
\mathbf{u}_{\mathrm{I}}=-\mathbf{1}_{\mathrm{x}} \mathrm{U}_{\mathrm{I}}
$$

in the sun-to-earth direction and carries an interplanetary magnetic field

$$
B_{I}=-\mathbf{1}_{z} B_{I}
$$

in the southward direction. In the remote region of undisturbed solar wind, the interplanetary electric field

$$
\mathbf{E}_{\mathrm{I}}=\mathbf{1}_{\mathrm{z}} \times \mathbf{1}_{\mathrm{x}} \mathrm{U}_{\mathrm{I}} \mathrm{B}_{\mathrm{I}}
$$

given by $-\mathbf{u}_{1} \times \mathbf{B}_{1}$ is in the dawn-to-dusk direction. We use rectangular coordinates $(x, z)$ for the noon-midnight plane, with the origin at the earth's center, the $x$-axis pointing sunward and the $\mathrm{z}$-axis pointing northward. We shall also use polar coordinates $(\mathrm{r}, \theta)$ with $\mathrm{x}=\mathrm{r} \sin \theta, \mathrm{z}=\mathrm{r} \cos \theta$, so the polar axis is along the z-axis, the noon plane has positive $\theta$ and the midnight plane has negative $\theta$. The solar wind will interact with the earth's dipole field. In the noon-midnight plane the earth's magnetic field will be approximated by 


$$
\mathbf{B}_{\mathrm{O}}(\mathbf{x}) \equiv-\mathrm{M}_{\mathrm{O}} \frac{\mathbf{1}_{\mathrm{x}} 2 \mathrm{xz}+\mathbf{1}_{\mathrm{z}}\left(-\mathrm{x}^{2}+\mathrm{z}^{2}\right)}{\left(\mathrm{x}^{2}+\mathrm{z}^{2}\right)^{2}}=-\mathrm{M}_{\mathrm{O}} \frac{\mathbf{1}_{\mathrm{r}} \cos \theta+\mathbf{1}_{\theta} \sin \theta}{\mathrm{r}^{2}},
$$

which is the magnetic field of a two-dimensional dipole with a southward moment of strength $\mathrm{M}_{\mathrm{O}}$.

The current induced in the interaction produces a perturbation magnetic field, to be denoted $\mathbf{B}_{\mathrm{P}}$, viz., $\mathbf{B}_{\mathrm{P}}^{(\mathrm{ext})}$ in the exterior region of interplanetary plasma and $\mathbf{B}_{\mathrm{P}}^{(\mathrm{int})}$ in the interior region of terrestrial plasma. With this large perturbation to the quasi-uniform interplanetary field and dipolar terrestrial field the resultant magnetic field

$$
\mathbf{B}(\mathbf{x})=\mathbf{B}_{\mathrm{I}}+\mathbf{B}_{\mathrm{O}}+\mathbf{B}_{\mathrm{P}}
$$

is the magnetospheric magnetic field. More precisely, the exterior field $\mathbf{B}^{(\text {ext })}=\mathbf{B}_{1}+\mathbf{B}_{\mathrm{P}}^{(\text {ext })}+\mathbf{B}_{0}$ is the magnetic field in the magnetosheath and the interior field $\mathbf{B}^{(\mathrm{int})}=\mathbf{B}_{\mathrm{O}}+\mathbf{B}_{\mathrm{P}}^{(\mathrm{int})}+\mathbf{B}_{1}$ is the magnetic field in the magnetosphere proper. The discontinuity of $\mathbf{B}_{\mathrm{P}}$ across the interface between the two regions accounts for the magnetopause current

$$
\mathbf{i}_{\mathrm{P}}=\mu_{0}^{-1} \mathbf{1}_{\mathrm{n}} \times\left(\mathbf{B}_{\mathrm{P}}^{(\mathrm{ext})}-\mathbf{B}_{\mathrm{P}}{ }^{(\mathrm{int})}\right) .
$$

Here $1_{n}$ denotes the unit vector outwardly normal to the magnetopause and $\mu_{0}$ is the magnetic permeability.

In terms of currents, the magnetospheric magnetic field is attributable to three currents. The earth's core current, which produces the dipole field $\mathbf{B}_{\mathrm{O}}$, has a dipole moment at the origin with other negative-order moments (such as quadrupole and octupole moments) being insignificant. The distant heliospherical current, which sustains the quasi-uniform field $\mathbf{B}_{\mathrm{I}}$, has a linear moment with other positive-order moments (such as quadratic and cubic moments) being insignificant. The induced magnetopause current, which produces the perturbation field $\mathbf{B}_{\mathrm{p}}$, has magnetic moments of various orders, whatever spatial distribution it may have. Although any current associated with field line reconnection is ignored, the occurrence of reconnection does affect $\mathbf{B}_{\mathrm{p}}$. In fact, open field lines that interconnect the earth and interplanetary space will show up in the superposition of the three constituent fields if the additive sum $\mathbf{B}_{\mathrm{I}}+\mathbf{B}_{\mathrm{O}}$ is not cancelled out by a part of $\mathbf{B}_{\mathrm{p}}$.

In the idealization that the induced magnetopause current is concentrated as a current sheet, the magnetospheric magnetic field is piecewise current-free. In the current-free (viz., $\nabla \times \mathbf{B}=\mathbf{0}$ by Ampere's law in differential form) regions the magnetospheric magnetic field has a scalar magnetic potential $\Omega(\mathbf{x})$, which is related to the magnetic field by $\mathbf{B}=-\nabla \Omega$. The magnetic potential is discontinuous at the points where the current is not zero. This is readily seen because the line integral of $\nabla \Omega$ will not be zero if the contour of integration encloses a current (by Ampere's law in integral form). The dipole field $\mathbf{B}_{\mathrm{o}}$ has the magnetic potential 


$$
\Omega_{\mathrm{O}}(\mathbf{x})=-\mathrm{M}_{\mathrm{O}} \frac{\mathrm{z}}{\mathrm{x}^{2}+\mathrm{z}^{2}}=-\mathrm{M}_{\mathrm{O}} \frac{\cos \theta}{\mathrm{r}},
$$

and the uniform field $\mathbf{B}_{1}$ has the magnetic potential

$$
\Omega_{\mathrm{I}}(\mathbf{x})=\mathrm{B}_{\mathrm{I} Z}=\mathrm{B}_{\mathrm{I}} \mathrm{r} \cos \theta .
$$

As to the induced field $\mathbf{B}_{\mathrm{p}}$, its magnetic potential $\Omega_{\mathrm{p}}$ is a piecewise harmonic function that vanishes at infinity and remains finite, say zero, at the origin. In the exterior region, $\Omega_{\mathrm{p}}$ has the multipole expansion

$$
\Omega_{\mathrm{P}}^{(\mathrm{ext})}(\mathbf{x})=\mathrm{c}_{-2} \omega^{(-2)}+\mathrm{c}_{-3} \omega^{(-3)}+\cdots,
$$

with certain coefficients $c_{-2}, c_{-3}, \ldots$, in terms of the two-dimensional harmonic functions of negative-order moment:

$$
\omega^{(-2)} \equiv \frac{\mathrm{z}}{\mathrm{x}^{2}+\mathrm{z}^{2}}=\frac{\cos \theta}{\mathrm{r}}, \quad \omega^{(-3)} \equiv \frac{\mathrm{xz}}{\left(\mathrm{x}^{2}+\mathrm{z}^{2}\right)^{2}}=\frac{\sin \theta \cos \theta}{\mathrm{r}^{2}}, \ldots
$$

On the other hand, in the interior region $\Omega_{\mathrm{p}}$ has the Taylor expansion

$$
\Omega_{\mathrm{P}}^{(\mathrm{int})}(\mathbf{x})=\mathrm{c}_{1} \omega^{(1)}+\mathrm{c}_{2} \omega^{(2)}+\cdots
$$

with matching coefficients $c_{1}, c_{2}, \ldots$, in terms of the two-dimensional harmonic functions of positive-order moment:

$$
\omega^{(1)} \equiv-\mathrm{z}=-\mathrm{r} \cos \theta, \quad \omega^{(2)} \equiv-\mathrm{xz}=-\mathrm{r}^{2} \sin \theta \cos \theta, \ldots .
$$

Note that $\omega^{(-2)}$ represents a positive (viz., northward) dipole, $\omega^{(-3)}$ a positive quardrupole, $\omega^{(1)}$ a positive (viz., northward) constant field, and $\omega^{(2)}$ a field with positive constant gradient. The crux of the problem is the induced magnetic field. Neither the geometric position of the magnetopause nor the spatial distribution of the magnetopause current is amenable to simple algorithms of calculation that involve considerations of force balance and flux continuity. Nevertheless, physically meaningful representations for $\mathbf{B}_{\mathrm{p}}$ can be devised by suitable prescriptions based on consideration of flux continuity alone. With $\Omega_{\mathrm{P}}^{(\mathrm{ext})}$ prescribed, by a suitable choice of the harmonic coefficients $c_{-2}, c_{-3}, \cdots$, the topological structure of $\mathbf{B}^{(e x t)}$ is readily ascertained by the neutral points and neutral line it contains. The magnetopause is then to be identified with a self-closed surface, in the external magnetic field $\mathbf{B}^{\text {(ext), that encloses }}$ the origin. The matching coefficients $c_{1}, c_{2}, \ldots$, for the internal magnetic field $\mathbf{B}^{(\text {int })}$ is to be determined by the continuity of magnetic flux through the magnetopause. Magnetic field lines are to be calculated by integration of the differential equation $\mathrm{d} \mathbf{x} / \mathrm{d} \tau=\mathbf{B}(\mathbf{x})$, with $\tau$ as a variable that specifies constituent points of a field line. Alternatively, we may prescribe the geometry of the magnetopause, both its closedness and openness parts. Then the exterior magnetic po- 
tential $\Omega^{\text {(ext) }}$, which becomes $\Omega_{1}$ at infinity, can be calculated by solving a Laplace equation subject to the boundary condition that $\Omega^{(\mathrm{ext})}$ has a zero normal derivative (viz., $\partial \Omega^{(\mathrm{ext})} / \partial \mathrm{n}=0$ ) on the prescribed closedness magnetopause and an invariant value (say, $\Omega^{(\mathrm{ext})}=0$ ) on the prescribed openness magnetopause. Correspondingly, the interior magnetic potential $\Omega^{(\mathrm{int})}$, which becomes $\Omega_{0}$ near the origin, can be calculated by solving a Laplace equation subject to the boundary condition that $\Omega^{(\mathrm{int})}$ has a normal derivative equal to the normal derivative of $\Omega^{\text {(ext) }}$

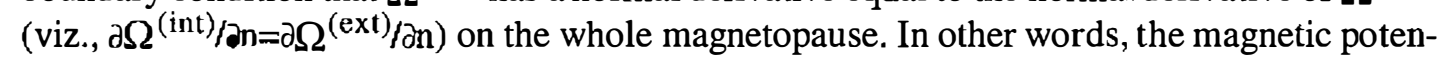
tial due to the magnetopause current can be determined by solving a Dirichlet-Neumann problem for $\Omega_{\mathrm{P}}^{(\mathrm{ext})}$ :

$$
\begin{aligned}
& \nabla^{2} \Omega_{\mathrm{P}}^{(\mathrm{ext})}=0,\left.\quad \Omega_{\mathrm{P}}^{(\mathrm{ext})}\right|_{\text {infinity }}=0, \\
& \left.\frac{\partial \Omega_{\mathrm{P}}^{(\mathrm{ext})}}{\partial \mathrm{n}}\right|_{\text {closedness }}=-\frac{\partial \Omega_{\mathrm{I}}}{\partial \mathrm{n}}-\frac{\partial \Omega_{\mathrm{O}}}{\partial \mathrm{n}},\left.\quad \Omega_{\mathrm{P}}^{(\mathrm{ext})}\right|_{\text {openness }}=-\Omega_{\mathrm{I}}-\Omega_{\mathrm{O}}
\end{aligned}
$$

and then solving a Neumann problem for $\Omega_{\mathrm{P}}^{\text {(int) }}$ :

$$
\nabla^{2} \Omega_{\mathrm{P}}^{(\mathrm{int})}=0,\left.\quad \Omega_{\mathrm{P}}^{(\mathrm{int})}\right|_{\text {origin }}=0,\left.\quad \frac{\partial \Omega_{\mathrm{P}}^{(\mathrm{int})}}{\partial \mathrm{n}}\right|_{\text {magnetopause }}=\frac{\partial \Omega_{\mathrm{P}}^{(\mathrm{ext})}}{\partial \mathrm{n}} .
$$

For instance, the prescription of $c_{-2}=M_{0}+B_{1} R^{2}, c_{-3}=0, c_{-4}=0, \cdots$ for $\Omega_{P}^{(e x t)}=\left(M_{0}+B_{1} R^{2}\right)$ $r^{-1} \cos \theta$ renders

$$
\Omega^{(\mathrm{ext})}=\mathrm{B}_{\mathrm{I}}\left(\mathrm{r}+\frac{\mathrm{R}^{2}}{\mathrm{r}}\right) \cos \theta,
$$

which has a radial derivative equal to zero on the whole circle $r=R$. The corresponding magnetic field

$$
\mathbf{B}^{(\mathrm{ext})}=\mathrm{B}_{\mathrm{I}}\left[\mathbf{1}_{\mathrm{r}}\left(-1+\frac{\mathrm{R}^{2}}{\mathrm{r}^{2}}\right) \cos \theta+\mathbf{1}_{\theta}\left(1+\frac{\mathrm{R}^{2}}{\mathrm{r}^{2}}\right) \sin \theta\right]
$$

has a pair of neutral points at $r=R, \theta=\left(\frac{1}{2} \pm \frac{1}{2}\right) \pi$. The boundary condition on that circle as a magnetopause in complete closedness is $\partial \Omega_{\mathrm{P}}^{(\mathrm{int})} / \partial \mathrm{r}=-\left(\mathrm{B}_{\mathrm{I}}+\mathrm{M}_{\mathrm{O}} / \mathrm{R}^{2}\right) \cos \theta$. The solution for the matching Neumann problem is $\Omega_{\mathrm{P}}^{(\text {int })}=-\left(\mathrm{B}_{\mathrm{I}}+\mathrm{M}_{\mathrm{O}} / \mathrm{R}^{2}\right) \mathbf{r} \cos \theta$. Namely, $\mathrm{c}_{1}=\mathrm{B}_{1}+\mathrm{M}_{\mathrm{o}} / \mathrm{R}^{2}, \mathrm{c}_{2}=0, \mathrm{c}_{3}=0$, 
.... Hence

$$
\Omega^{(\text {int })}=M_{O}\left(-\frac{1}{r}-\frac{r}{R^{2}}\right) \cos \theta
$$

and

$$
\mathbf{B}^{(\mathrm{int})}=\mathrm{M}_{\mathrm{O}}\left[\mathbf{1}_{\mathrm{r}}\left(-\frac{1}{\mathrm{r}^{2}}+\frac{1}{\mathrm{R}^{2}}\right) \cos \theta+\mathbf{1}_{\theta}\left(-\frac{1}{\mathrm{r}^{2}}-\frac{1}{\mathrm{R}^{2}}\right) \sin \theta\right] .
$$

It follows from $\mathbf{B}_{\mathrm{P}}^{\text {(ext) }}=-\left(1+\mathrm{B}_{\mathrm{I}} \mathrm{R}^{2} / \mathrm{M}_{\mathrm{O}}\right) \mathbf{B}_{\mathrm{O}}$ and $\mathbf{B}_{\mathrm{P}}^{(\mathrm{int})}=-\left(1+\mathrm{M}_{\mathrm{O}} / \mathrm{B}_{\mathrm{I}} \mathrm{R}^{2}\right) \mathbf{B}_{\mathrm{I}}$ that the magnetopause current $i_{P}=1_{z} \times 1_{x} \mu_{0}^{-1}\left(2 B_{1}+2 M_{o} / R^{2}\right) \sin \theta$ is equivalent to a dipole of northward moment $M_{O}+B_{I} R^{2}$ at the origin as far as the exterior magnetic field is concerned and is equivalent to a current at infinity that sustains a northward constant field of strength $B_{1}+M_{O} / R^{2}$ as far as the interior magnetic field is concerned. As the radius $R$ varies from 0 to $\infty$, the dipole moment $M_{O}+B_{I} R^{2}$ increases from $M_{0}$ to $+\infty$ and the linear moment $B_{1}+M_{0} / R^{2}$ decreases from $+\infty$ to $B_{I}$. The choice of $R=\left(M_{O} / B_{\mathrm{I}}\right)^{1 / 2}$ will yield the prototype closed magnetosphere given by $B^{(e x t)}=B_{I}-B_{O}$ and $\mathbf{B}^{(\mathrm{int})}=\mathbf{B}_{\mathrm{O}}-\mathbf{B}_{\mathrm{r}}$. The magnetopause current is $\mathbf{1}_{\mathrm{z}} \times \mathbf{1}_{\mathrm{x}} 4 \mu_{0}{ }^{-1} \mathrm{~B}_{\mathrm{I}} \sin \theta$.

On the other hand, if a circle of radius $\mathrm{R}$ centered at the origin is prescribed as the magnetopause in full openness, the solution of the Dirichlet-Neumann problem is

$$
\Omega^{(\mathrm{ext})}=\mathrm{B}_{\mathrm{I}}\left(\mathrm{r}-\frac{\mathrm{R}^{2}}{\mathrm{r}}\right) \cos \theta
$$

which has a value equal to zero on the whole circle $r=R$. Thus $\Omega_{P}^{(\text {ext })}=\left(M_{0}-B_{I} R^{2}\right) r^{-1} \cos \theta$. The magnetic field

$$
\mathbf{B}^{(\mathrm{ext})}=\mathrm{B}_{\mathrm{I}}\left[\mathbf{1}_{\mathrm{r}}\left(-1-\frac{\mathrm{R}^{2}}{\mathrm{r}^{2}}\right) \cos \theta+\mathbf{1}_{\theta}\left(1-\frac{\mathrm{R}^{2}}{\mathrm{r}^{2}}\right) \sin \theta\right]
$$

vanishes at $r=R, \theta= \pm \frac{1}{2} \pi$. Since $\partial \Omega^{(e x t)} / \partial r$ has the value of $2 B_{I} \cos \theta$ on the circular magnetopause in full openness, the solution for the matching Neumann problem is

$$
\Omega^{(\text {int })}=\left[M_{O}\left(-\frac{1}{r}-\frac{r}{R^{2}}\right)+2 B_{1} r\right] \cos \theta
$$

Thus $\Omega_{\mathrm{P}}^{(\text {int })}=\left(\mathrm{B}_{\mathrm{I}}-\mathrm{M}_{\mathrm{O}} / \mathrm{R}^{2}\right) \mathrm{r} \cos \theta$. The corresponding magnetic field

$$
\mathbf{B}^{(\mathrm{int})}=\mathbf{1}_{\mathrm{r}}\left[\mathrm{M}_{\mathrm{O}}\left(-\frac{1}{\mathrm{r}^{2}}+\frac{1}{\mathrm{R}^{2}}\right)-2 \mathrm{~B}_{\mathrm{I}}\right] \cos \theta+\mathbf{1}_{\theta}\left[-\mathrm{M}_{\mathrm{O}}\left(\frac{1}{\mathrm{r}^{2}}+\frac{1}{\mathrm{R}^{2}}\right)+2 \mathrm{~B}_{\mathrm{I}}\right] \sin \theta
$$


has a poloidal component in addition to the required radial component on the circle $r=R$. The discontinuity in the poloidal magnetic field incurs a magnetopause current, which can be rather small. It follows from $\mathbf{B}_{\mathrm{P}}^{(\mathrm{ext})}=\left(-1+\mathrm{B}_{\mathrm{I}} \mathrm{R}^{2} / \mathrm{M}_{\mathrm{O}}\right) \mathbf{B}_{\mathrm{O}}$ and $\mathbf{B}_{\mathrm{P}}^{(\mathrm{int})}=\left(1-\mathrm{M}_{\mathrm{O}} / \mathrm{B}_{\mathrm{r}} \mathrm{R}^{2}\right) \mathbf{B}_{\mathrm{r}}$ that the magnetopause current $i_{P}=\mathbf{1}_{z} \times \mathbf{1}_{x} \mu_{0}{ }^{-1}\left(2 M_{O} / R^{2}-2 B_{I}\right) \sin \theta$ has an internal image, for the exterior magnetic field, that consists of a dipole of moment $\mathbf{l}_{z}\left(M_{O}-B_{I} R^{2}\right)$ at the origin and an extemal image, for the interior magnetic field, at infinity that sustains a constant field $\mathbf{1}_{z}\left(\mathrm{M}_{\mathrm{O}} / \mathrm{R}^{2}-\mathrm{B}_{\mathrm{I}}\right)$. As the radius $R$ varies from 0 to $\infty$, the dipole moment $M_{O}-B_{1} R^{2}$ decreases from $M_{O}$ to $-\infty$ and the linear moment $M_{0} / R^{2}-B_{1}$ decreases from $+\infty$ to $-B_{1}$. The choice of $R=\left(M_{0} / B_{1}\right)^{1 / 2}$ will yield the prototype open magnetosphere given by $\mathbf{B}=\mathbf{B}_{\mathrm{I}}+\mathbf{B}_{\mathrm{O}}$ everywhere. The magnetopause current happens to be zero.

\section{MAGNETOSPHERIC ELECTRIC FIELD}

The steady-state electric field $\mathbf{E}(\mathbf{x})$ in the magnetosphere is curl-free (viz., $\nabla \times \mathbf{E}=\mathbf{0}$ by Faraday's law). Thus, it has an electric potential $\Phi(\mathbf{x})$, which is related to the electric field by $\mathbf{E}=-\nabla \Phi$. In the remote region of the $x z$-plane $\mathbf{E}$ is in the direction perpendicular to the plane. There $\Phi$ has the same asymptotic value, say zero.

With a perfect electrical conductivity for space plasma the sum $\mathbf{E}+\mathbf{u} \times \mathbf{B}$, which signifies the electric field in a local coordinate frame that moves with the plasma's flow velocity $\mathbf{u}$, is zero. Namely,

$$
\mathbf{E}=-\mathbf{u} \times \mathbf{B}
$$

By virtue of $\mathbf{B} \cdot \mathbf{E}=0$, the electric field has no component aligned with the magnetic field. It follows from $(\mathbf{B} \cdot \nabla) \Phi=0$ that the electric potential is invariant on individual magnetic field lines. Hence the electric potential remains to have the value of zero on all magnetic field lines, in the noon-midnight plane, that map from infinity. So the part of the xz-plane in the exterior region is an electric equipotential surface. There the electric field is perpendicular to the $\mathrm{xz}$ plane unless $\mathbf{B}$ becomes zero to nullify the direction of $\mathbf{E}$.

\section{DRIFT MOTION OF SOLAR WIND PLASMA}

The bulk motion of a plasma in a magnetic field can be described by a flow velocity $\mathbf{u}=\mathbf{u}_{\perp}+\mathbf{u}_{\|}$that comprises a cross-field drift velocity and a field-aligned slide velocity. It is the drift motion that carries frozen-in magnetic field lines to cross magnetic separatrices in entering or exiting magnetic cells of different field line connectivity. Thus, we may neglect the slide motion, which passively accommodates the continuity of mass flow.

The drift velocity

$$
\mathbf{u}_{\perp}=\frac{\mathbf{E} \times \mathbf{B}}{\mathbf{B} \cdot \mathbf{B}}
$$


is perpendicular to both the electric and the magnetic fields. The perpendicularity means that the drift velocity vector lies on an electric equipotential surface and also on a magnetic equipotential surface. Hence, various intersection curves of electric and magnetic equipotential surfaces are streamlines of drift motion. Moreover, each electric equipotential surface is covered by various streamlines, so is each magnetic equipotential surface. Accordingly, the xz-traces of various magnetic equipotential surfaces are streamlines in the noon-midnight plane of zero electric potential.

More important are the solar wind streamlines on the magnetopause. The streamlines with zero magnetic potential constitute the openness magnetopause. As to the streamlines on the closedness magnetopause, they are non-planar curves of zero electric potential. These streamlines with zero electric potential have xz-trace points. The trace points appear as sink or source of mass and flux flows in our two-dimensional treatment.

\section{MAGNETOPAUSE}

The earth's magnetic field deflects the oncoming solar wind. So the interplanetary plasma, carrying the frozen-in interplanetary magnetic field lines, flows around the earth at some distances. The various points of closest approach to the earth constitute a self-closed surface that encloses all terrestrial plasma. This surface, separating interplanetary and terrestrial plasmas, is the magnetopause in entirety. In other words, the magnetopause comprises points where the interplanetary plasma makes contact with the terrestrial plasma. These contact points of interplanetary plasma are grouped into segments of solar wind streamlines. Some of the streamline segments are on an electric equipotential surface. Others are on a magnetic equipotential surface. The former solar wind streamlines cover the exterior face of the current sheet which is sandwiched by interplanetary and terrestrial field lines. The latter solar wind streamlines run from a site of field line reconnection to another site. Open field lines are formed by reconnection between interplanetary and closed field lines approaching to each other. At subsequent reconnection between opposite segments of open field lines interplanetary and closed field lines are reverted back.

The part of magnetopause with an invariant electric potential, passed through by electric field lines, is also covered by magnetic field lines in perpendicularity to the streamlines. On the closedness magnetopause the solar wind streamlines carry interplanetary field lines that slip on the exterior face of the magnetopause current sheet. On the other hand, the part of magnetopause with an invariant magnetic potential, passed through by magnetic field lines, is also covered by electric field lines in perpendicularity to the streamlines. On the openness magnetopause the solar wind streamlines carry open field lines that traverse the magnetopause.

When the openness part of the magnetopause is non-existent (as if shrinking to the antisolar point) so the whole magnetopause is in closedness, the magnetosphere is completely closed. In the other limit, when the closedness part of the magnetopause is non-existent (as if shrinking to the subsolar point) so the whole magnetopause is in openness, the magnetosphere is fully open. 


\section{PROTOTYPE CLOSED MAGNETOSPHERE}

Before we deal with partially open magnetospheres, it is instructive to examine two prototype magnetospheres. One of them is completely closed, with $\Omega_{\mathrm{P}}{ }^{\text {(ext) }}=-2 \Omega_{\mathrm{O}}$ and $\Omega_{\mathrm{P}}^{\text {(int) }}=$ $-2 \Omega_{\mathrm{l}}$. The other is fully open, with $\Omega_{\mathrm{p}}=0$. Both of them have a circular magnetopause, as the xz-trace of a corresponding spherical magnetopause in three-dimensional setting.

First, we consider the prototype closed magnetosphere. It has the magnetic potential

$$
\begin{array}{r}
\Omega^{(\text {ext })}=\left(\mathrm{B}_{\mathrm{I}} \mathrm{r}+\frac{\mathrm{M}_{\mathrm{O}}}{\mathrm{r}}\right) \cos \theta, \\
\Omega^{(\mathrm{int})}=\left(-\frac{\mathrm{M}_{\mathrm{O}}}{\mathrm{r}}-\mathrm{B}_{\mathrm{I}} \mathrm{r}\right) \cos \theta .
\end{array}
$$

Both exterior and interior magnetic potentials have a normal derivative of zero on the circle $\mathrm{r}=$ $\left(\mathrm{M}_{\mathrm{o}} / \mathrm{B}_{\mathrm{I}}\right)^{1 / 2}$. The circle, without magnetic flux passing through, will be shown the magnetopause. It is an electric equipotential curve that encloses the earth at the origin. The magnetic field

$$
\begin{gathered}
\mathbf{B}^{(\mathrm{ext})}=\mathbf{1}_{\mathrm{r}}\left(-\mathrm{B}_{\mathrm{I}}+\frac{\mathrm{M}_{\mathrm{O}}}{\mathrm{r}^{2}}\right) \cos \theta+\mathbf{1}_{\theta}\left(\mathrm{B}_{\mathrm{I}}+\frac{\mathrm{M}_{\mathrm{O}}}{\mathrm{r}^{2}}\right) \sin \theta, \\
\mathbf{B}^{(\mathrm{int})}=\mathbf{1}_{\mathrm{r}}\left(-\frac{\mathrm{M}_{\mathrm{O}}}{\mathrm{r}^{2}}+\mathrm{B}_{\mathrm{I}}\right) \cos \theta+\mathbf{1}_{\theta}\left(-\frac{\mathrm{M}_{\mathrm{O}}}{\mathrm{r}^{2}}-\mathrm{B}_{\mathrm{I}}\right) \sin \theta
\end{gathered}
$$

is symmetric about the $\mathrm{z}$-axis. The exterior magnetic field has a pair of neutral points at $\mathrm{z}=$ $\pm\left(M_{0} / B_{1}\right)^{1 / 2}$ of the $z$-axis on the exterior face of the current sheet, which is covered by semicircular magnetic field lines that emanate from the north neutral point and terminate at the south neutral point. The interior magnetic field has a pair of neutral points at the pole points $\mathrm{z}= \pm\left(\mathrm{M}_{\mathrm{o}} / \mathrm{B}_{\mathrm{J}}\right)^{1 / 2}$ of the $\mathrm{z}$-axis on the interior face of the current sheet, which is covered by semicircular magnetic field lines that emanate from the south neutral point and terminate at the north neutral point. These magnetic field lines have the same electric potential as the neutral points. The magnetic neutral points on the exterior and interior faces of current sheet are Ttype in local field-line topology. The flux function

$$
\begin{aligned}
& \Psi^{(\text {ext })}=\left(-B_{I} r+\frac{M_{0}}{r}\right) \sin \theta \\
& \Psi(\text { int })=\left(-\frac{M_{O}}{r}+B_{I} r\right) \sin \theta
\end{aligned}
$$

given by the integral $\int_{0}^{\theta} B_{r}(r, \dot{\theta}) r d \dot{\theta}$ for the magnetic flux is invariant on individual magnetic 
field lines. The two semi-circular field lines, emanating from the north neutral point and terminate at the south neutral point, have an electric potential of zero. They constitute a self-closed curve that encloses the origin. The whole plane is partitioned by the magnetopause into two magnetic cells of interplanetary or closed field lines. There is no cell of open field lines. The two cells share the magnetopause as a common wall, which is a current sheet. The magnetopause current, being sandwiched by $\mathbf{B}^{(\mathrm{ext})}=\mathbf{1}_{\theta} 2 \mathrm{~B}_{1} \sin \theta$ on the exterior face and $\mathbf{B}^{\text {(int) }}=-\mathbf{1}_{\theta} 2 \mathrm{~B}_{I} \sin \theta$ on the interior face, is $\mathbf{i}_{\mathrm{P}}=\mathbf{1}_{z} \times \mathbf{1}_{\mathrm{x}} 4 \mu_{0}^{-1} \mathrm{~B}_{\mathrm{I}} \sin \theta$. There is no magnetic equipotential curve enclosing the origin.

Figure 2 shows the flow of solar wind that passes around the magnetopause. The solar wind streamlines, delineated by magnetic equipotential lines $\left[1+\mathrm{M}_{\mathrm{o}} / \mathrm{B}_{\mathrm{I}}\left(\mathrm{x}^{2}+\mathrm{z}^{2}\right)\right] \mathrm{z}=\mathrm{Z}_{\mathrm{c}}$, are orthogonal to the magnetic field lines $\left[1-\mathrm{M}_{0} / \mathrm{B}_{1}\left(\mathrm{x}^{2}+\mathrm{z}^{2}\right)\right] \mathrm{x}=\mathrm{x}_{\infty}$. The solar wind does not flow across the circle of zero electric potential at all. This electric equipotential curve interfaces between interplanetary and terrestrial plasmas. It constitutes the magnetopause of the completely closed magnetosphere. Upon incidence on the magnetopause in the noon plane the solar wind stream-

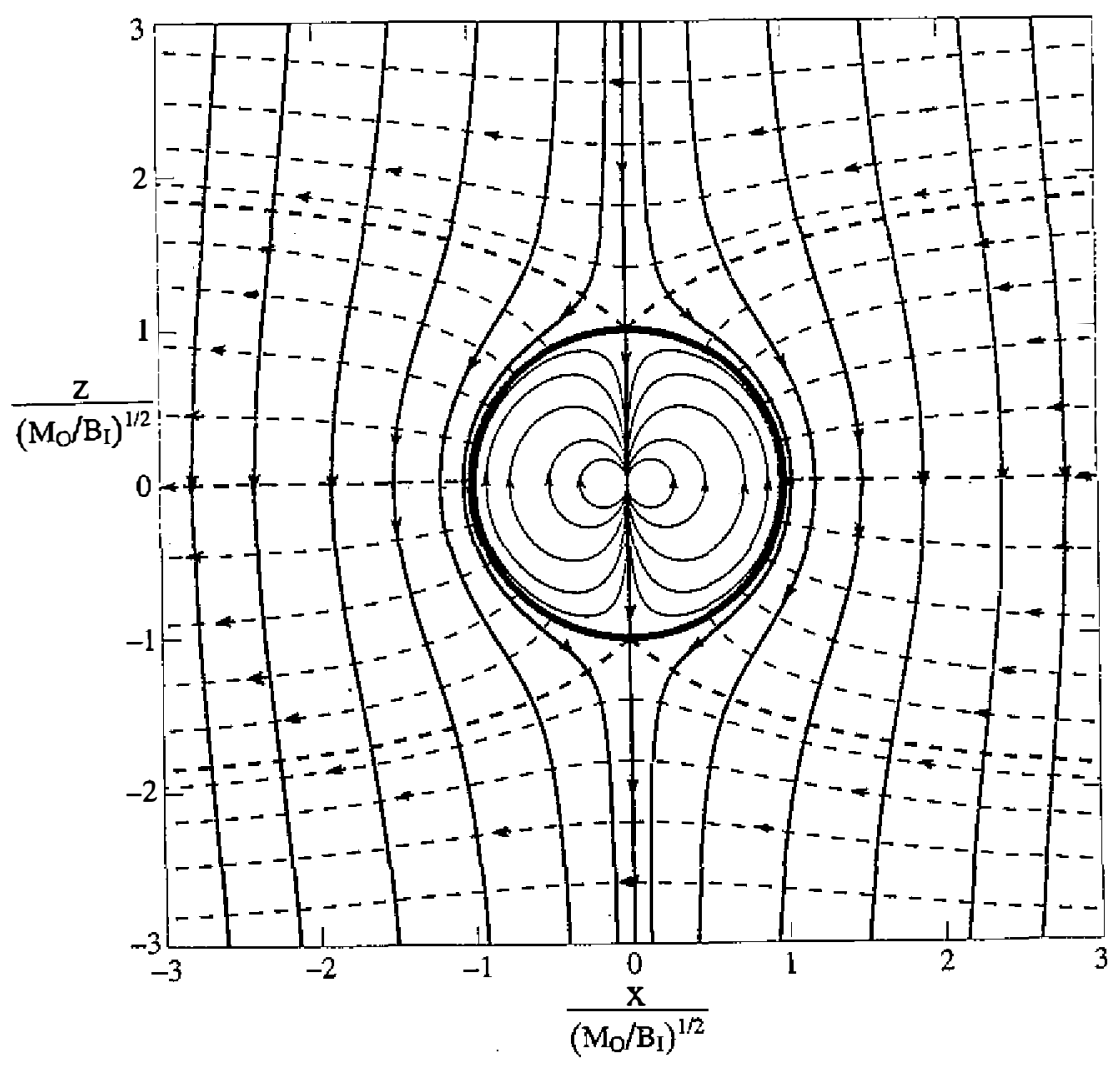

Fig. 2. Prototype closed magnetosphere with circular magnetopause, represented by $\Omega^{\text {(ext) }}=\Omega_{\mathrm{I}}-\Omega_{\mathrm{O}}$ and $\Omega^{(\mathrm{int})}=\Omega_{\mathrm{O}}-\Omega_{\mathrm{I}}$. Solid lines are magnetic field lines. Dashed lines are solar wind streamlines. 
lines split to leave the noon plane. The diverted streamlines, staying on the magnetopause in three-dimensional setting, become confluent to leave the magnetopause in the midnight plane (cf. Fig. 1a). Hence, in our two-dimensional treatment the dayside magnetopause appears as a sink of mass and flux flows and the nightside magnetopause a source. The subsolar point of this closedness magnetopause is at $\mathrm{x}=\left(\mathrm{M}_{\mathrm{O}} / \mathrm{B}_{\mathrm{I}}\right)^{1 / 2}$ on the positive $\mathrm{x}$-axis and the antisolar point is at $x=-\left(M_{0} / B_{I}\right)^{1 / 2}$ on the negative $x$-axis.

\section{PROTOTYPE OPEN MAGNETOSPHERE}

Next, we consider the prototype open magnetosphere. It has the magnetic potential

$$
\Omega=\left(\mathrm{B}_{\mathrm{I}} \mathrm{r}-\frac{\mathrm{M}_{\mathrm{O}}}{\mathrm{r}}\right) \cos \theta,
$$

in both the exterior and interior regions. This magnetic potential has a value of zero on the circle $\mathrm{r}=\left(\mathrm{M}_{\mathrm{O}} / \mathrm{B}_{\mathrm{l}}\right)^{1 / 2}$ as well as on the equatorial line $\mathrm{z}=0$. The circle, with magnetic flux passing through, will be shown the magnetopause. It is a magnetic equipotential curve that encloses the earth at the origin. The magnetic field

$$
B=\mathbf{1}_{\mathrm{r}}\left(-\mathrm{B}_{\mathrm{I}}-\frac{\mathrm{M}_{\mathrm{O}}}{\mathrm{r}^{2}}\right) \cos \theta+\mathbf{1}_{\theta}\left(\mathrm{B}_{\mathrm{I}}-\frac{\mathrm{M}_{\mathrm{O}}}{\mathrm{r}^{2}}\right) \sin \theta
$$

is symmetric about the z-axis. It vanishes on the intersection of the circular and straight lines of zero magnetic potential. The two intersection points $r=\left(M_{\mathrm{O}} / \mathrm{B}_{1}\right)^{1 / 2}, \theta= \pm \frac{1}{2} \pi$ are the $x z$-trace of a magnetic neutral line in the three-dimensional setting. The flux function is

$$
\Psi=\left(-\mathrm{B}_{\mathrm{I}} \mathrm{r}-\frac{\mathrm{M}_{\mathrm{O}}}{\mathrm{r}}\right) \sin \theta .
$$

The whole plane is partitioned by a two-branch separatrix $\left(\mathrm{B}_{\mathrm{I}} \mathrm{r}+\mathrm{M}_{\mathrm{O}} / \mathrm{r}\right) \sin \theta=2 \mathrm{~B}_{1}^{1 / 2} \mathrm{M}_{\mathrm{o}}^{1 / 2}$ into three magnetic cells, which are filled with closed, open, or detached interplanetary field lines, respectively. The three cells make contact at the cross points of magnetic neural line, which are $\mathrm{X}$-type in local field line topology. The magnetic field is radial, equal to $-\mathbf{1}_{\mathrm{r}} 2 \mathrm{~B}_{1} \cos \theta$, at the magnetopause, through which open field lines pass. The magnetopause current happens to be zero because $\mathbf{B}_{\mathrm{p}}$ is zero. There is no electric equipotential curve enclosing the origin.

Figure 3 shows the flow of solar wind that passes around the magnetopause. The solar wind streamlines, delineated by magnetic equipotential lines $\left[1-\mathrm{M}_{\mathrm{o}} / \mathrm{B}_{1}\left(\mathrm{x}^{2}+\mathrm{z}^{2}\right)\right] \mathrm{z}=\mathrm{z}_{\infty}$, are orthogonal to the magnetic field lines $\left[1+\mathrm{M}_{\mathrm{O}} / \mathrm{B}_{\mathrm{I}}\left(\mathrm{x}^{2}+\mathrm{z}^{2}\right)\right] \mathrm{x}=\mathrm{x}_{\infty}$. The solar wind does not flow across the circle of zero magneic potential at all. This magnetic equipotential curve interfaces between interplanetary and terrestrial plasmas. It constitutes the magnetopause of the fully open magnetosphere. Upon incidence at the subsolar point $\mathrm{x}=\left(\mathrm{M}_{\mathrm{O}} / \mathrm{B}_{\mathrm{r}}\right)^{1 / 2}$ on the positive $\mathrm{x}$-axis the solar wind streamlines split to leave the equatorial line. The diverted streamlines, staying on the magnetopause in the noon-midnight plane, become confluent to leave the magneto- 
pause at the antisolar point $x=-\left(M_{o} / B_{1}\right)^{1 / 2}$ on the negative $x$-axis (cf. Fig. 1b).

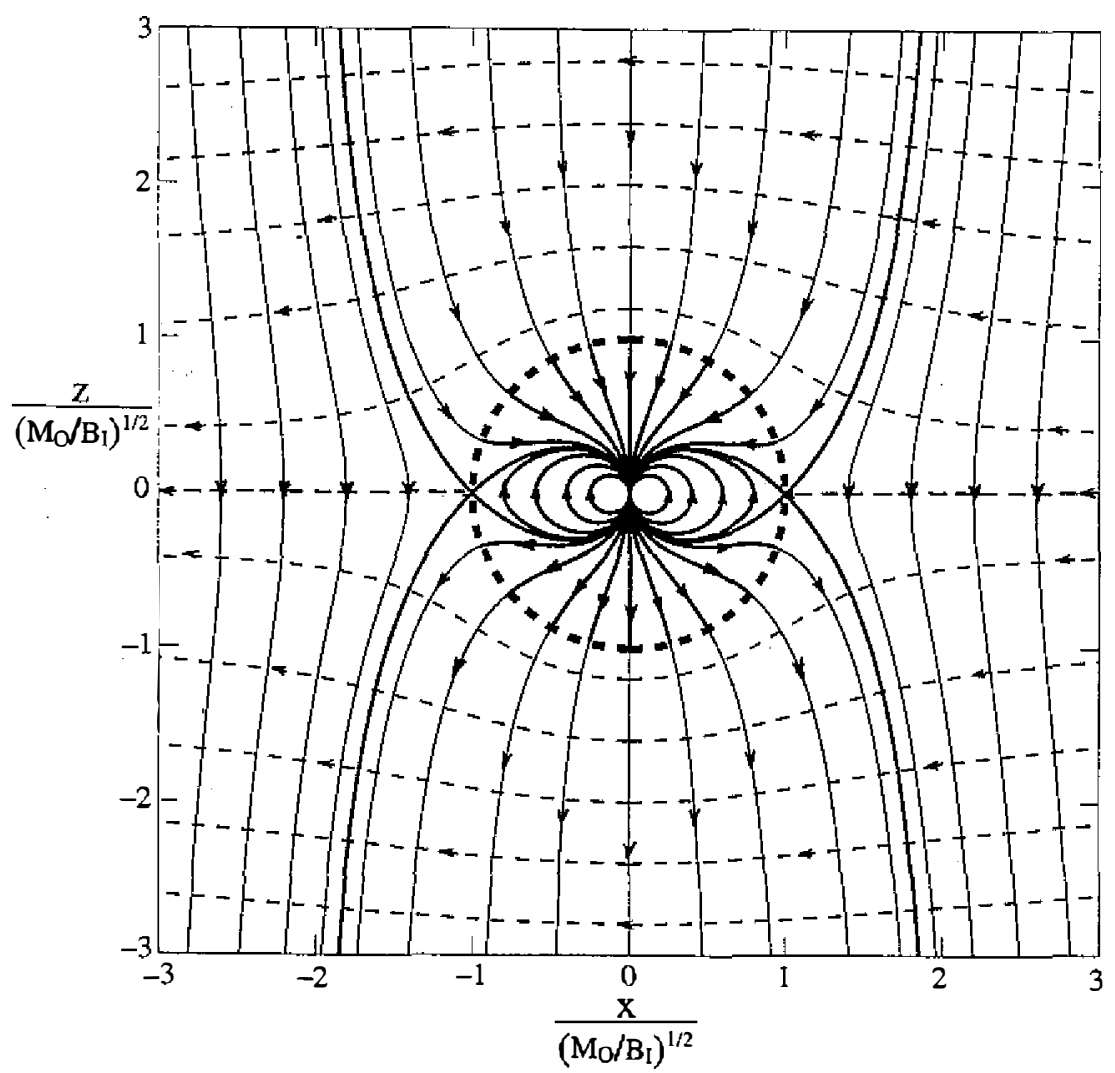

Fig. 3. Prototype open magnetosphere with circular magnetopause, represented by $\Omega=\Omega_{\mathrm{I}}+\Omega_{\mathrm{o}}$. Solid lines are magnetic field lines. Dashed lines are solar wind streamlines.

\section{CLOSED OR OPEN MAGNETOSPHERES WITH NON-CIRCULAR MAGNETO- PAUSE}

The configurations of magnetic field lines and solar wind streamlines for the two prototype magnetospheres hold, with suitable scalings, even for other completely closed or fully open magnetospheres with circular magnetopause. This is so because upon replacement of $M_{0}$ by $B_{I} R^{2}$ equation (23) become equation (13) and upon replacement of $B_{1}$ by $M_{0} / R^{2}$ equation (24) become equation (15) whereas upon replacement of $M_{0}$ by $B_{1} R^{2}$ equation (29) becomes equation (17) and upon replacement of $B_{I}$ by $2 B_{I}-M_{o} / R^{2}$ equation (29) becomes equation (19). In these configurations the circular magnetopause has a geometry of high symmetry. So the exterior and interior harmonic functions due to the magnetopause current are simple terms with a magnetic moment of the lowest order. The coefficient $c_{-2}$, equal to $M_{0}+B_{1} R^{2}$ in the case of closed magnetosphere or equal to $\mathrm{M}_{0}-\mathrm{B}_{\mathrm{I}} \mathrm{R}^{2}$ in the case of open magnetosphere, for the 
dipole moment ranges between $-\infty$ and $+\infty$ when the free parameter $\mathrm{R}$ for the radius of circle varies from 0 to $\infty$. And the coefficient $c_{1}$, equal to $B_{I}+M_{0} / R^{2}$ in the case of closed magnetosphere or equal to $-B_{I}+M_{O} / R^{2}$ in the case of open magnetosphere, for the linear moment ranges between $-B_{1}$ and $+\infty$ when the free parameter $R$ varies from 0 to $\infty$. Accordingly, if $c_{1}$ is less than $-\mathrm{B}_{\mathrm{I}}$, the magnetopause can not be circular in either complete closedness or full openness. In that case, the harmonic function due to the magnetopause current must have additional terms of higher order and the magnetopause is non-circular.

To examine closed or open magnetospheres with a non-circular magnetopause, we consider

$$
\Omega^{(e x t)}=B_{I} r \cos \theta+\left(-M_{O}+c_{-2}\right) \frac{\cos \theta}{r}+c_{-3} \frac{\sin \theta \cos \theta}{r^{2}}
$$

which contains only dipole and quadrupole moments in the internal image of the magnetopause current. The magnetic potential is zero on the curve $c_{-3} \sin \theta=-B_{I} r^{3}+\left(M_{O}-c_{-2}\right) r$. It will be self-closed to enclose the origin if $c_{-2}$ is less than $M_{0}$ and $c_{-3}$ has a magnitude less than the maximal value $2\left(\mathrm{M}_{\mathrm{O}}-\mathrm{c}_{-2}\right)^{3 / 2} / 3^{3 / 2} \mathrm{~B}_{\mathrm{I}}^{1 / 2}$. This self-closed curve of zero magnetic potential is a non-circular magnetopause of a fully open magnetosphere. The exterior magnetic field

$$
\mathbf{B}^{(\mathrm{ext})}=\mathrm{B}_{\mathrm{I}}\left(-\mathbf{1}_{\mathrm{r}} \cos \theta+\mathbf{1}_{\theta} \sin \theta\right)+\left(-\mathrm{M}_{\mathrm{O}}+\mathrm{c}_{-2}\right) \frac{\mathbf{1}_{\mathrm{r}} \cos \theta+\mathbf{1}_{\theta} \sin \theta}{\mathrm{r}^{2}}+\mathrm{c}_{-3} \frac{\mathbf{1}_{\mathrm{r}} \sin 2 \theta-\mathbf{1}_{\theta} \cos 2 \theta}{\mathrm{r}^{3}}
$$

may have a pair of neutral points at where the curve $2\left(c_{-2}-M_{0}\right) r=c_{-3}(1 / \sin \theta-4 \sin \theta)$ of vanishing $\mathbf{1}_{x} \cdot \mathbf{B}^{\text {(ext) }}$ intersects the curve $2 \mathrm{~B}_{\mathrm{I}} \mathrm{r}^{3} \sin \theta=\mathrm{c}_{-3}$ which is obtained from the vanishing of $\mathbf{1}_{2} \cdot \mathbf{B}^{\text {(ext) }}$ upon the use of $\mathbf{1}_{x} \cdot \mathbf{B}^{\text {(ext) }}$ being zero. The curve of zero electric potential, formed by the magnetic field lines that emanate from the north neutral point and terminate at the south neutral point, will be self-closed to enclose the origin if $c_{-2}$ is greater than $M_{0}$ and $c_{-3}$ has a magnitude not too large. This self-closed curve of zero electric potential is a non-circular magnetopause of a completely closed magnetosphere.

Otherwise, if $c_{-3}$ has a sufficiently large magnitude, neither the curve of zero magnetic potential nor the curve of zero electric potential will be self-closed to enclose the origin. So the magnetopause is neither in full openness nor in complete closedness. In other words, the magnetosphere has to be partially open.

\section{PARTIALLY OPEN MAGNETOSPHERES}

The magnetopause of a partially open magnetosphere comprises a part with an invariant electric equipotential in the front and a part with an invariant magnetic equipotential in the rear. The front part is formed by the field lines that emanate from the north magnetic neutral point and/or terminate at the south magnetic neutral point. The rear part contains points of magnetic neutral line. The front and rear parts seamed together enclose the origin. An example of partially open magnetosphere is one having a circular magnetopause with its front half in 
closedness and its rear half in openness.

To see the features of a partially open magnetosphere with a non-circular magnetopause we shall examine the harmonic expansion

$$
\Omega^{(\mathrm{ext})}=\mathrm{B}_{\mathrm{I}} \mathrm{Z}+\left(-\mathrm{M}_{\mathrm{O}}+\mathrm{c}_{-2}\right) \frac{\mathrm{z}}{\mathrm{x}^{2}+\mathrm{z}^{2}}+\mathrm{c}_{-3} \frac{\mathrm{xz}}{\left(\mathrm{x}^{2}+\mathrm{z}^{2}\right)^{2}}+\cdots
$$

and its magnetic field

$$
\mathbf{B}^{(\mathrm{ext})}=-\mathbf{1}_{\mathrm{z}} \mathrm{B}_{\mathrm{I}}+\left(-\mathrm{M}_{\mathrm{O}}+\mathrm{c}_{-2}\right) \frac{\mathbf{1}_{\mathrm{x}} 2 \mathrm{xz}+\mathbf{1}_{\mathrm{z}}\left(-\mathrm{x}^{2}+\mathrm{z}^{2}\right)}{\left(\mathrm{x}^{2}+\mathrm{z}^{2}\right)^{2}}+\mathrm{c}_{-3} \frac{\mathbf{1}_{\mathrm{x}}\left(3 \mathrm{x}^{2} \mathrm{z}-\mathrm{z}^{3}\right)+\mathbf{1}_{\mathrm{z}}\left(-\mathrm{x}^{3}+3 \mathrm{xz}\right)}{\left(\mathrm{x}^{2}+\mathrm{z}^{2}\right)^{3}}+\cdots
$$

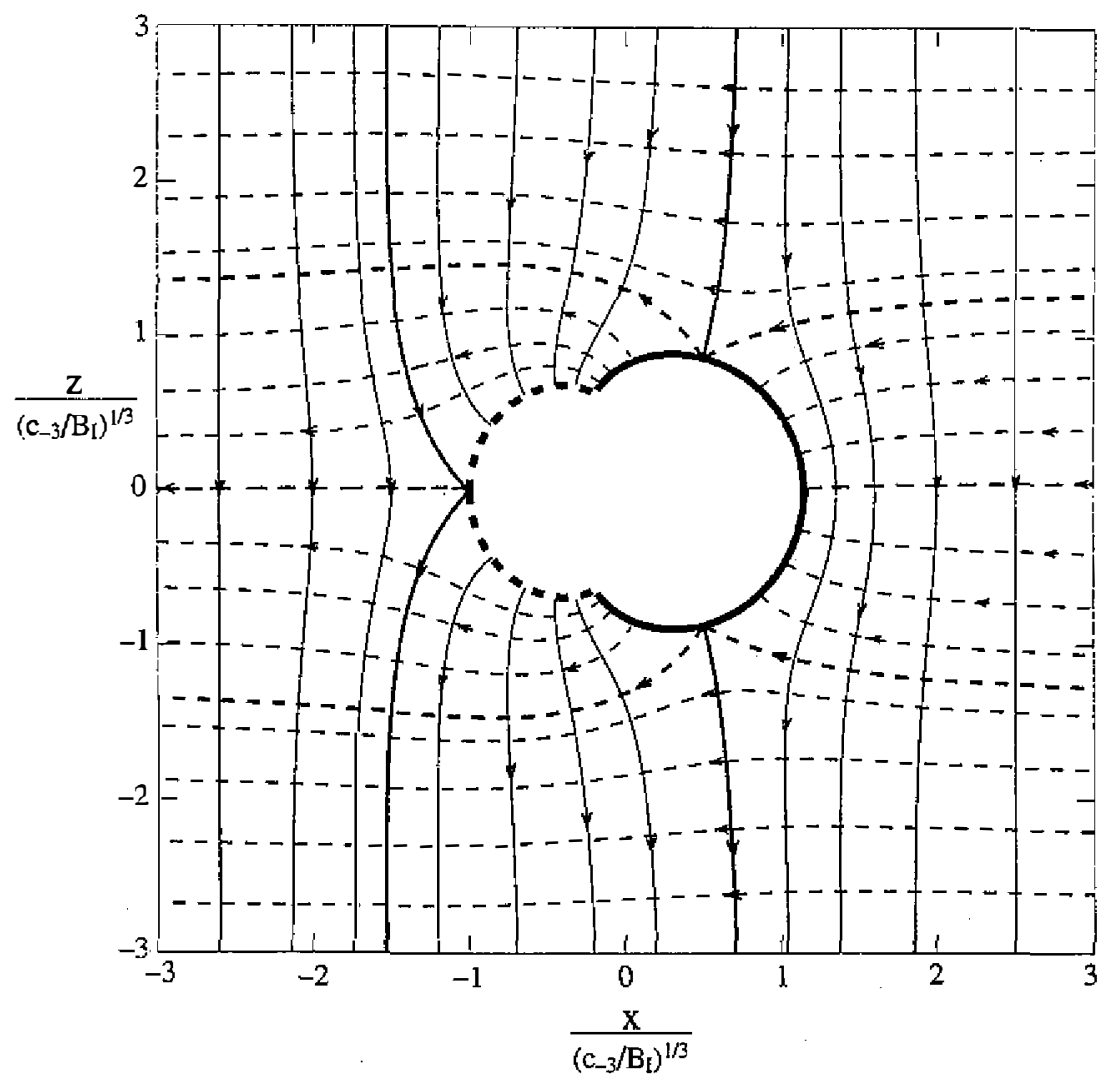

Fig. 4. A southward uniform field immersing a positive quadrupole, represented by $\Omega^{(e x t)}=B_{1} z+c_{-3} x z /\left(x^{2}+z^{2}\right)^{2}$. Thick solid line is curve of zero electric potential and thick dashed line is curve of zero magnetic potential. Solid lines are magnetic field lines. Dashed lines are solar wind streamlines. 
In the previous section we have discussed the case of $\mathrm{c}_{-4}, \mathrm{c}_{-5}, \cdots$ all being zero. When $\mathrm{c}_{-3}$ is positive and sufficiently large, the curve of zero electric potential will appear in the front and the curve of zero magnetic potential in the rear. The two curves intersect at a pair of points. The front part of the electric equipotential curve and the rear part of the magnetic equipotential curve together form a closed curve that encloses the origin. With $\mathrm{c}_{-2}=\mathrm{M}_{0}$ and $\mathrm{c}_{-3}>0$, the configuration for $\Omega^{(\mathrm{ext})}=\Omega_{\mathrm{I}}+\mathrm{c}_{-3} \omega^{(-3)}$ is as shown in Fig. 4 . The cross point of neural line is at $\mathrm{x}=-\left(\mathrm{c}_{-3} / \mathrm{B}_{\mathrm{I}}\right)^{1 / 3}$ on the negative $\mathrm{x}$-axis and the neutral points are at $\mathrm{x}=\frac{1}{2}\left(\mathrm{c}_{-3} / \mathrm{B}_{\mathrm{I}}\right)^{1 / 3}, \mathrm{z}= \pm \frac{1}{2} \sqrt{3}$ $\left(\mathrm{C}_{-3} / \mathrm{B}_{\mathrm{I}}\right)^{1 / 3}$. This configuration amounts to a southward uniform field immersing a positive quadrupole. Again, in our two-dimensional treatment, the middle part of the closedness magnetopause appears as a sink of mass and flux flows and the remaining part as a source.

More interesting is the case with $c_{-2}=M_{D}, c_{-3}=2 M_{D} x_{D}, \cdots$ so that the series, in positive powers of $\mathrm{x}_{\mathrm{D}}$, for $\Omega_{\mathrm{P}}^{\text {(ext) }}$ converges to $\mathrm{z} /\left[\left(\mathrm{x}-\mathrm{x}_{\mathrm{D}}\right)^{2}+\mathrm{z}^{2}\right]$. In this case the multipole expansion (34) sums to $\Omega_{1}+\Omega_{0}+\Omega_{\mathrm{D}}$, with

$$
\Omega_{D} \equiv M_{D} \frac{z}{\left(x-x_{D}\right)^{2}+z^{2}}
$$

signifying a northward image dipole of moment $M_{D}$ at $x=x_{D}$ on the positive $x$-axis. The offorigin dipole has various moments of negative order at the origin. Consider $\mathbf{M}_{\mathrm{D}}$ to be greater than $\mathrm{M}_{\mathrm{O}}$ so that the northward image dipole will overpower the earth's southward dipole. With

$$
\mathbf{B}_{\mathrm{D}} \equiv \mathrm{M}_{\mathrm{D}} \frac{\mathbf{1}_{\mathrm{x}} 2\left(\mathrm{x}-\mathrm{x}_{\mathrm{D}}\right) \mathrm{z}+\mathbf{1}_{\mathrm{z}}\left[-\left(\mathrm{x}-\mathrm{x}_{\mathrm{D}}\right)^{2}+\mathrm{z}^{2}\right]}{\left[\left(\mathrm{x}-\mathrm{x}_{\mathrm{D}}\right)^{2}+\mathrm{z}^{2}\right]^{2}},
$$

the magnetic field $\mathbf{B}^{(\mathrm{ext})}=\mathbf{B}_{\mathrm{I}}+\mathbf{B}_{\mathrm{O}}+\mathbf{B}_{\mathrm{D}}$ has a pair of neutral points and a cross point of neutral line. The neutral points are at $\mathrm{x}=\mathrm{x}_{0}, \mathrm{z}= \pm \mathrm{f}_{\mathrm{D}}\left(\mathrm{x}_{0}\right)$, with $\mathrm{x}_{0}$ satisfying the equation

$$
\mathrm{M}_{\mathrm{O}} \frac{\mathrm{x}^{2}-\mathrm{z}^{2}}{\left(\mathrm{x}^{2}+\mathrm{z}^{2}\right)^{2}}-\left.\mathrm{M}_{\mathrm{D}} \frac{\left(\mathrm{x}-\mathrm{x}_{\mathrm{D}}\right)^{2}-\mathrm{z}^{2}}{\left[\left(\mathrm{x}-\mathrm{x}_{\mathrm{D}}\right)^{2}+\mathrm{z}^{2}\right]^{2}}\right|_{\mathrm{z}=\mathrm{f}_{\mathrm{D}}(\mathrm{x})}=\mathrm{B}_{\mathrm{I}}
$$

in the interval $x_{D}<x<x_{D} /\left(1-M_{O} / M_{D}\right)$. Here the function

$$
f_{D}\left(x ; x_{D}, M_{D} / M_{O}\right) \equiv\left\{\frac{\left[\left(M_{D} / M_{O}\right)\left(x-x_{D}\right) / x\right]^{1 / 2} x^{2}-\left(x-x_{D}\right)^{2}}{1-\left[\left(M_{D} / M_{O}\right)\left(x-x_{D}\right) / x\right]^{1 / 2}}\right\}^{1 / 2},
$$

signifying the distance from the $\mathrm{x}$-axis for the curve of vanishing $\mathbf{1}_{\mathrm{x}} \cdot\left(\mathbf{B}_{\mathrm{o}}+\mathbf{B}_{\mathrm{D}}\right)$, is real-valued in the interval $x_{D} \leq x \leq x_{D} /\left(1-M_{o} / M_{D}\right)$ and also in the interval $-x_{D} /\left[\left(M_{D} / M_{O}\right)^{1 / 3}-1\right] \leq x<0$. The cross point of neutral line is on the negative $x$-axis, with an $x$-coordinate satisfying the equation 


$$
\frac{\mathrm{M}_{\mathrm{O}}}{\mathrm{x}^{2}}-\frac{\mathrm{M}_{\mathrm{D}}}{\left(\mathrm{x}-\mathrm{x}_{\mathrm{D}}\right)^{2}}=\mathrm{B}_{\mathrm{I}}
$$

in the interval $\left.-x_{D} /\left[M_{D} / M_{O}\right)^{1 / 3}-1\right] \leq x<0$. Figure 5 shows the obtained configuration.

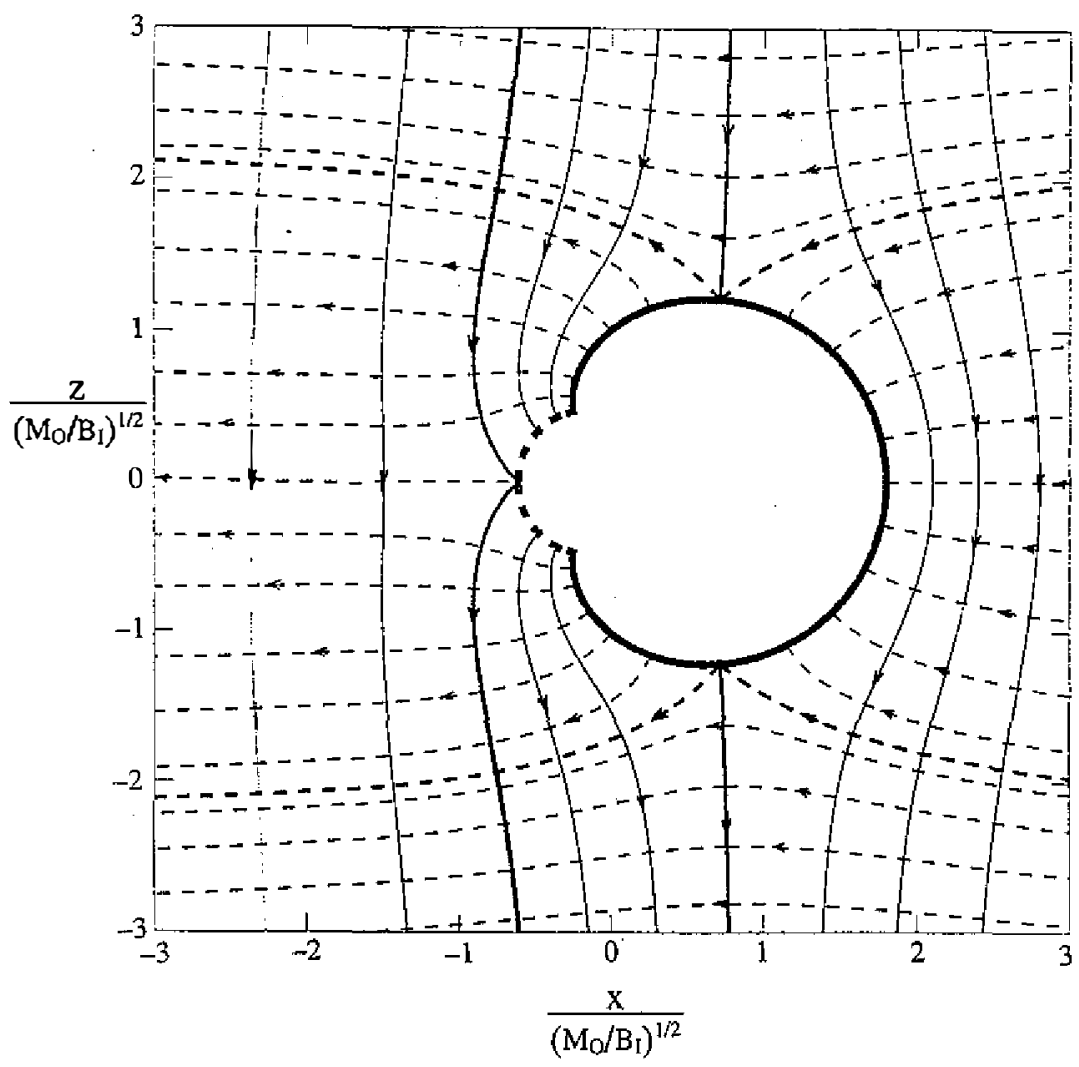

Fig. 5. Exterior configuration of a partially open magnetosphere, represented by $\Omega^{(\text {int })}=\Omega_{\mathrm{T}}+\Omega_{\mathrm{O}}+\Omega_{\mathrm{D}}$ with $\mathrm{M}_{\mathrm{D}} / \mathrm{M}_{\mathrm{O}}=2$ and $\mathrm{x}_{\mathrm{D}} /\left(\mathrm{M}_{\mathrm{O}} / \mathrm{B}_{\mathrm{I}}\right)^{1 / 2}=0.5$. Thick solid line is closedness magnetopause and thick dashed line is openness magnetopause. Solid lines are magnetic field lines. Dashed lines are solar wind streamlines.

In lieu of solving a Neumann problem, the matching $\Omega_{\mathrm{P}}^{(\mathrm{int})}$ may be approximated by

$$
\Omega_{C} \equiv-M_{C} \frac{z}{\left(x-x_{C}\right)^{2}+z^{2}}
$$

upon optimal choice of the free parameters $M_{C}$ and $x_{C}$. The magnetic potential $\Omega_{C}$ signifies a southward image dipole of moment $M_{C}$ at $x=x_{C}$ on the positive $x$-axis. By virtue of the series 
$z /\left[\left(x-x_{C}\right)^{2}+z^{2}\right] \equiv z / x_{C}^{2}+2 x z / x_{C}^{3}+\cdots$ in negative powers of $x_{C}$, the off-origin dipole $\Omega_{C}$ has various positive moments $c_{1}=M_{C} / x_{C}^{2}, c_{2}=2 M_{C} / x_{C}^{3}, \cdots$ of positive order at infinity. With

$$
\mathbf{B}_{C} \equiv-\mathbf{M}_{C} \frac{\mathbf{1}_{\mathrm{x}} 2\left(\mathrm{x}-\mathrm{x}_{\mathrm{C}}\right) \mathrm{z}+\mathbf{1}_{\mathrm{z}}\left[-\left(\mathrm{x}-\mathrm{x}_{\mathrm{C}}\right)^{2}+\mathrm{z}^{2}\right]}{\left[\left(\mathrm{x}-\mathrm{x}_{\mathrm{C}}\right)^{2}+\mathrm{z}^{2}\right]^{2}},
$$

the magnetic field $\mathbf{B}^{(\mathrm{int})}=\mathbf{B}_{\mathrm{O}}+\mathbf{B}_{1}+\mathbf{B}_{\mathrm{C}}$ has a pair of neutral points and a cross point of neutral line when $M_{C}$ is greater than $M_{O}\left(c f\right.$. Yeh 1997). The neutral points are at $x=x_{0}, z= \pm f_{c}\left(x_{0}\right)$, with $\mathrm{x}_{0}$ satisfying the equation

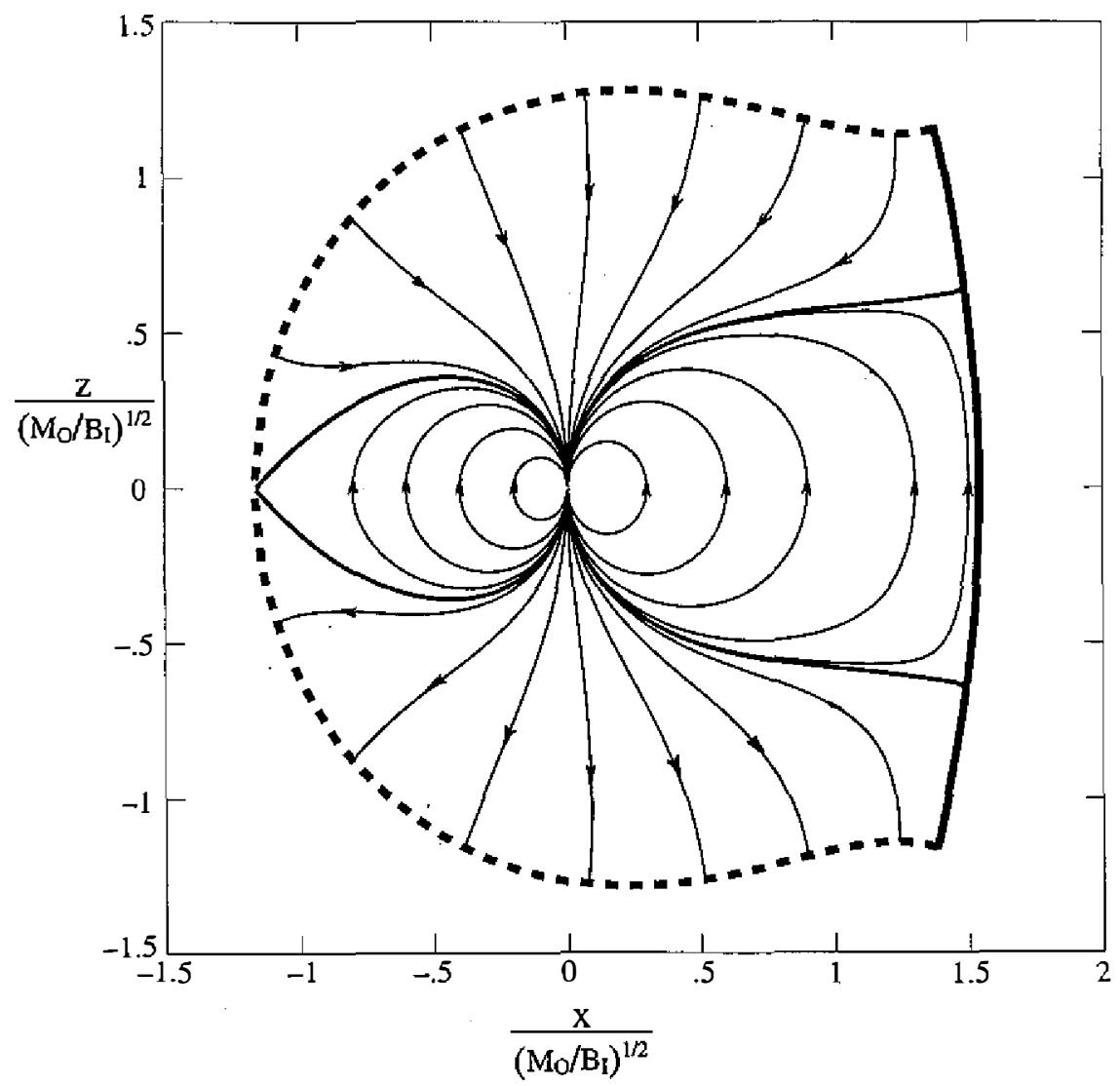

Fig. 6. Interior configuration of a partially open magnetosphere, represented by $\Omega^{(i n t)}=\Omega_{\mathrm{O}}+\Omega_{\mathrm{I}}+\Omega_{\mathrm{C}}$ with $\mathrm{M}_{\mathrm{C}} / \mathrm{M}_{\mathrm{O}}=10$ and $\mathrm{x}_{\mathrm{C}} /\left(\mathrm{M}_{\mathrm{d}} / \mathrm{B}_{\mathrm{I}}\right)^{1 / 2}=5$. Thick solid line is closedness magnetopause and thick dashed line is openness magnetopause. Solid lines are magnetic field lines. Dashed lines are solar wind streamlines. 


$$
\mathrm{M}_{\mathrm{O}} \frac{\mathrm{x}^{2}-\mathrm{z}^{2}}{\left(\mathrm{x}^{2}+\mathrm{z}^{2}\right)^{2}}+\left.\mathrm{M}_{\mathrm{C}} \frac{\left(\mathrm{x}-\mathrm{x}_{\mathrm{C}}\right)^{2}-\mathrm{z}^{2}}{\left[\left(\mathrm{x}-\mathrm{x}_{\mathrm{C}}\right)^{2}+\mathrm{z}^{2}\right]^{2}}\right|_{\mathrm{z}=\mathrm{f}_{\mathrm{C}}(\mathrm{x})}=\mathrm{B}_{\mathrm{I}}
$$

in the interval $0<x<x_{c} /\left[1+\left(M_{C} / M_{O}\right)^{1 / 3}\right]$. The cross point of neutral line is on the negative $x$ axis, with an $\mathrm{x}$-coordinate satisfying the equation

$$
\frac{\mathrm{M}_{\mathrm{O}}}{\mathrm{x}^{2}}+\frac{\mathrm{M}_{\mathrm{C}}}{\left(\mathrm{x}-\mathrm{x}_{\mathrm{C}}\right)^{2}}=\mathrm{B}_{\mathrm{I}}
$$

Here the function

$$
\mathrm{f}_{\mathrm{C}}\left(\mathrm{x} ; \mathrm{x}_{\mathrm{C}}, \mathrm{M}_{\mathrm{C}} / \mathrm{M}_{\mathrm{O}}\right) \equiv\left\{\frac{\left(\mathrm{x}_{\mathrm{C}}-\mathrm{x}\right)^{2}-\left[\left(\mathrm{M}_{\mathrm{C}} / \mathrm{M}_{\mathrm{O}}\right)\left(\mathrm{x}_{\mathrm{C}}-\mathrm{x}\right) / \mathrm{x}\right]^{1 / 2} \mathrm{x}^{2}}{\left[\left(\mathrm{M}_{\mathrm{C}} / \mathrm{M}_{\mathrm{O}}\right)\left(\mathrm{x}_{\mathrm{C}}-\mathrm{x}\right) / \mathrm{x}\right]^{1 / 2}-1}\right\}^{1 / 2}
$$

is real-valued in the interval $0<x_{C}<x_{C}\left[1+\left(M_{C} / M_{O}\right)^{1 / 3}\right]$ and also in the interval $x_{C} /\left(1+M_{o} / M_{C}\right)$ $<\mathrm{x}<\mathrm{x}_{\mathrm{C}}$. Figure 6 shows the obtained configuration. The pertinent feature is seen in the magnetic potential

$$
\Omega^{(i n t)}=-M_{O} \frac{\cos \theta}{r}+\left(B_{1}-c_{1}\right) r \cos \theta-c_{2} r^{2} \sin \theta \cos \theta .
$$

The curve of zero magnetic potential is $c_{2} \sin \theta=-M_{O} / r^{3}+\left(B_{I}-c_{1}\right) / r$. It will be a self-closed curve enclosing the origin when $c_{1}$ is less than $B_{I}$ and $c_{2}$ has a magnitude less than the maximal value $2\left(\mathrm{~B}_{\mathrm{I}}-\mathrm{c}_{1}\right)^{3 / 2} / 3^{3 / 2} \mathrm{M}_{\mathrm{O}}^{1 / 2}$. The magnetic field

$$
\mathbf{B}^{(\mathrm{int})}=-\mathrm{M}_{\mathrm{O}} \frac{\mathbf{1}_{\mathrm{x}} 2 \mathrm{xz}+\mathbf{1}_{\mathrm{z}}\left(-\mathrm{x}^{2}+\mathrm{z}^{2}\right)}{\left(\mathrm{x}^{2}+\mathrm{z}^{2}\right)^{2}}+\mathbf{1}_{\mathrm{z}}\left(-\mathrm{B}_{\mathrm{I}}+\mathrm{c}_{1}\right)+2 \mathrm{c}_{2}\left(\mathbf{1}_{\mathrm{x}} \mathrm{z}+\mathbf{1}_{\mathrm{z}} \mathrm{x}\right)
$$

has a pair of neutral points at where the curve $2 \mathrm{M}_{\mathrm{O}} \sin \theta / \mathrm{r}^{3}=\mathrm{c}_{2}$ of vanishing $\mathbf{1}_{\mathrm{x}} \cdot \mathbf{B}^{\text {(int) }}$ intersects the curve $\left(c_{1}-B_{1}\right) r^{2}=M_{0}\left(1-4 \sin ^{2} \theta\right)$. With $c_{1}=B_{1}$ and $c_{2}>0$, the configuration for $\Omega^{(\text {int })}=\Omega_{0}+c_{2} \omega^{(2)}$ is as shown in Fig. 7. The cross point of neutral line is at $x=-\left(M_{\circ} / c_{2}\right)^{1 / 3}$ on the negative $\dot{x}$-axis and the neutral points are at $x=\frac{1}{2}\left(M_{0} / c_{2}\right)^{1 / 3}, z= \pm \frac{1}{2} \sqrt{3}\left(M_{0} / c_{2}\right)^{1 / 3}$. This configuration amounts to a southward dipole being immersed in a magnetic field that has an $\mathrm{x}$-component proportional to the distance from the $\mathrm{x}$-axis and a $\mathrm{z}$-component proportional to the distance from the $\mathrm{z}$-axis.

\section{DISCUSSION}

The magnetosphere formed in the interaction between the earth's magnetic field and the magnetized solar wind is not unique in terms of the earth's dipole and the interplanetary mag- 


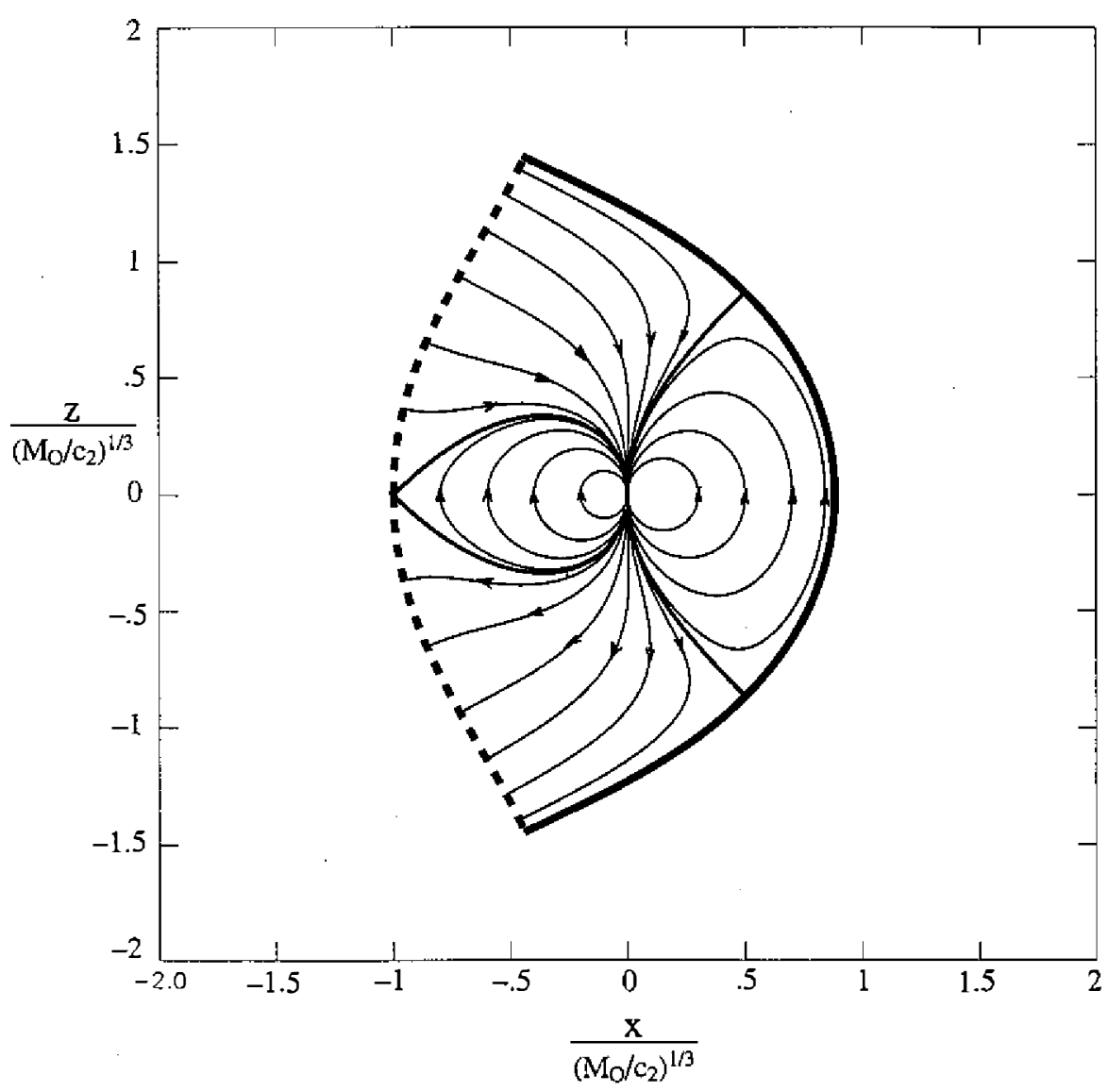

Fig. 7. A southward dipole immersed in a non-uniform magnetic field, represented by $\Omega^{(i n t)}=-M_{0} z /\left(x^{2}+z^{2}\right)-c_{2} x z$. Thick solid line is curve of zero electric equipotential and thick dashed line is curve of zero magnetic potential. Solid lines are magnetic field lines. Dashed lines are solar wind streamlines.

netic field. It can be closed, open, or partially open, depending on the magnetopause current induced in the interaction. So are the size and shape of the magnetopause, depending on the induced current. For a magnetosphere to be completely closed, the magnetopause current must have a positive (viz., northward) dipole moment large enough to overpower the earth's negative (viz., southward) dipole moment and also have a positive linear moment large enough to overpower the negative linear moment of the interplanetary magnetic field. The larger the magnetopause current, the smaller the size of the closedness magnetopause will be. On the other hand, for a magnetosphere to be fully open, the magnetopause current can not have a positive dipole moment too large as to overpower the earth's negative dipole moment. An eastward magnetopause current will make the size of the openness magnetopause large whereas a westward magnetopause current will make it small. 
For a magnetosphere to be partially open, the magnetopause current must have a northward dipole moment large enough to overpower the earth's southward dipole and a linear moment not too large so the southward interplanetary field is not overpowered. Moreover, to keep the closedness part of the magnetopause in the front and the openness part in the rear, the quadrupole and quadratic moments of the magnetopause current must be positive. Thus, the exterior magnetic field is somewhat like that of the interplanetary field immersing a positive quadrupole and the interior magnetic field is somewhat like that of the earth's dipole being immersed in a magnetic field of positive quadratic moment. Accordingly, as far as the magnetic field in the exterior region is concerned, the magnetopause current is somewhat like an off-origin northward dipole placed inside the self-closed surface of the magnetopause. And as far as the magnetic field in the interior region is concerned, the magnetopause current is somewhat like a southward dipole placed outside the magnetopause, on the sunward side.

Finally, we mention the usage of complex variables to facilitate the calculations of neural points, magnetic field lines and solar wind streamlines in two-dimenstional configurations. In terms of the complex variable $Z \equiv \mathrm{x}-i \mathrm{z}$, the complex field $B(Z) \equiv \mathrm{B}_{\mathrm{z}}(\mathrm{x}, \mathrm{z})+i \mathrm{~B}_{\mathrm{x}}(\mathrm{x}, \mathrm{z})$ is related to the complex potential $\Phi(Z) \equiv \Psi(\mathrm{x}, \mathrm{z})+i \Omega(\mathrm{x}, \mathrm{z})$ by $B=-\mathrm{d} \Phi / \mathrm{d} Z$ (see Yeh 1999$)$. The uniform interplanetary field is represented by the potential $\Phi_{\mathrm{I}} \equiv \mathrm{B}_{\mathrm{I}} Z$ and the earth' dipole field by $\Phi_{\mathrm{O}} \equiv$ $\mathrm{M}_{\mathrm{O}} Z$. The magnetic field due to the magnetopause current is represented by the Laurent expansion

$$
\Phi_{P}^{(\mathrm{ext})}=-\mathrm{c}_{-2} \frac{1}{Z}-\frac{1}{2} \mathrm{c}_{-3} \frac{1}{Z^{2}}-\cdots
$$

in the exterior region and by the Taylor expansion

$$
\Phi_{P}^{(\text {int })}=-\mathrm{c}_{1} Z-\frac{1}{2} \mathrm{c}_{2} Z^{2}-\cdots
$$

in the interior region. The choice of $c_{-2}=M_{D}, c_{-3}=2 M_{D} x_{D}, \cdots$ yields $\Phi_{P}^{(e x t)}=-M_{D} /\left(Z-x_{D}\right)$ and the approximation of $\mathrm{c}_{1} \approx \mathrm{M}_{C} / \mathrm{x}_{C}^{2}, \mathrm{c}_{2} \approx 2 \mathrm{M}_{\mathrm{C}} / \mathrm{x}_{C}{ }_{C}, \cdots$ yields $\Phi_{P}^{(\mathrm{int})} \approx \mathrm{M}_{C} /\left(Z-\mathrm{x}_{\mathrm{C}}\right)+\mathrm{M}_{\mathrm{C}} / \mathrm{x}_{\mathrm{C}}$. Following the computation algorithm for the interior magnetic field $B^{(\text {int })} \approx M_{O} / Z^{2}-B_{I}+M_{C} /\left(Z-x_{C}\right)^{2}(Y e h$, 2000), the magnetic neutral points and cross point of neutral line in the exterior magnetic field $B^{(\mathrm{ext})}=-\mathrm{B}_{\mathrm{I}}+\mathrm{M}_{\mathrm{O}} / Z^{2}-\mathrm{M}_{\mathrm{D}} /\left(Z-\mathrm{x}_{\mathrm{D}}\right)^{2}$ can be computed straightforwardly by solving the quartic equation

$$
\mathrm{B}_{\mathrm{I}} Z^{4}-2 \mathrm{~B}_{\mathrm{I}} \mathrm{x}_{\mathrm{D}} Z^{3}+\left(\mathrm{B}_{\mathrm{I}} \mathrm{x}_{\mathrm{D}}^{2}-\mathrm{M}_{\mathrm{O}}+\mathrm{M}_{\mathrm{D}}\right) Z^{2}+2 \mathrm{M}_{\mathrm{O}} \mathrm{x}_{\mathrm{D}} Z-\mathrm{M}_{\mathrm{O}} \mathrm{x}_{\mathrm{D}}^{2}=0
$$

using the classical Ferrari's method. And following the algorithm for the interior potential $\Phi^{(\text {int })} \approx \mathrm{M}_{\alpha} / Z+\mathrm{B}_{\mathrm{I}} Z+\mathrm{M}_{\mathrm{C}} /\left(Z-\mathrm{x}_{\mathrm{C}}\right)+\mathrm{M}_{\mathrm{C}} / \mathrm{x}_{\mathrm{C}^{\prime}}$ the equipotential lines and field lines associated with the exterior potential $\Phi^{(\text {ext })}=\mathrm{B}_{\mathrm{I}} Z+\mathrm{M}_{\mathrm{O}} / Z-\mathrm{M}_{\mathrm{D}} /\left(Z-\mathrm{x}_{\mathrm{D}}\right)$ can be computed by solving the cubic equation 


$$
\mathrm{B}_{\mathrm{I}} Z^{3}-\left(\mathrm{B}_{\mathrm{I}} \mathrm{x}_{\mathrm{D}}+\Phi\right) Z^{2}+\left(\mathrm{M}_{\mathrm{O}}-\mathrm{M}_{\mathrm{D}}+\mathrm{x}_{\mathrm{D}} \Phi\right) Z-\mathrm{M}_{\mathrm{O}} \mathrm{x}_{\mathrm{D}}=0
$$

using the classical Cardano's method. Field lines are given by the inverse function $Z(\Phi)$ with $\Omega$ as a varying parameter. And streamlines are given by $Z(\Phi)$ with $\Psi$ as a varying parameter.

Acknowledgments This research was supported by the National Science Council under grant NSC 89-2111-M-008-018 to the National Central University.

\section{REFERENCES}

Cahill, L. J. Jr., 1995: Early studies of the boundary of the geomagnetic field: a historical review. Physics of the Magnetopause. In: P. Song, B.U.Ö. Sonnerup and M. F. Thomsen (Eds.). Geophys. Monog. Ser., 90, 9-15, AGU, Washington, D. C.

Chapman, S., 1963: Solar plasma, geomagnetism and aurora. Geophysics-The Earth's Environment. In: C. DeWitt, J. Hieblot, and A. Lebeau (Eds.), 372-502, Gordon and Breach Science Publishers, New York.

Dungey, J. W., 1961: Interplanetary magnetic field and the auroral zones. Phys. Rev. Lett., 6, $47-48$.

Ness, N. F., 1987: Magnetotail research: the early years. Magnetotail Physics. In: A. T. Y. Lui (Ed.), p. 11, Johns Hopkins University Press, Baltimore.

Siscoe, G. L., 1988: The magnetospheric boundary. Physics of Space Plasmas (1987). In: T. Chang, G. B. Crew and J. R. Jasperse (Eds.), 3-78, Scientific Publishers, Cambridge, Massachusetts.

Yeh, T., 1997: Magnetospheric structure with a southward interplanetary magnetic field. $J$. Geophys. Res., 102, 51-56.

Yeh, T., 1999: General solution of two-dimensional Chapman-Ferraro problem of magnetospheric cavity, TAO, 10, 397-414.

Yeh, T., 2000: Magnetospheric configuration with magnetotail current, TAO, 11, 423-438. 